11. This research was performed while Dr. Glader was a recipient of a Research Career Development Award AM-00435 from the National Institutes of Health. Support also was provided by NIH Grant AM-20879.

12. We wish to thank Margaret Macksey for technical assistance and Luella Walter

for secretarial assistance in the preparation of this manuscript.
13. Address all correspondence to Dr. Bertil E. Glader, Division of Hematology/ Oncology, Children's Hospital at Stanford, 520 Willow Road, Palo Alto, CA 94304.

14. Received for publication December 14, 1983.

\title{
The Current Status of Auditory Brainstem Response Testing in Neonatal Populations
}

\author{
L. CLARKE COX ${ }^{(50)}$ \\ Department of Audiology, Cleveland State University, Cleveland, Ohio, USA
}

\begin{abstract}
Summary
The use of auditory brainstem response (ABR) for assessment of hearing in the neonate has not been without challenge. Although numerous articles have appeared, agreement regarding the utility of neonatal ABR testing does not exist. In review of the current studies and commentaries, a clear majority are favorable to neonatal ABR testing. These studies along with current test procedures are discussed.
\end{abstract}

\section{Abbreviations}

ABR, auditory brainstem response NICU, neonatal intensive care unit COG-Crib-O-Gram

Since the initial reporting of ABR in 1970 by Jewett et al. (26), the potential of accurate evaluation of auditory function in the neonate has existed. With the subsequent reporting of use in the NICU in 1975 by Schulman-Galambos and Galambos (38), procedures for the assessment of that potential were initiated. Since 1975, the research reported in the literature has grown exponentially each year. In spite of the large and increasing amount of research reported, severl issues have remained unresolved, viz., the applicability, reliability, and validity of neonatal ABR testing. In fact, because of these and other factors, some authors have begun to seriously question using ABR with neonates $(13,35,42)$.

The need for early identification of infants with hearing loss is apparent. Normal language, learning, and social skill acquisition is contingent upon hearing. Although the incidence of hearing impairment in the general neonatal population is relatively low $(0.26 \%)(41)$, in NICU neonates the incidence is considerably higher $(2-10 \%)(16,39)$. Furthermore, the improved survival rate of neonates born weighing less than $1500 \mathrm{~g}(19,31)$ has increased the pool of infants in which a high incidence of hearing impairment is seen (16).

Because the need for early identification of hearing loss does exist and the current procedures that could identify hearing impairment in the neonate are open to question (ABR), a critical review of $A B R$ testing is in order. The purpose of this article is to summarize the current ABR literature, both pro and con, and to draw conclusions regarding its use based on the literature and the experience of the writer. The summary will not attempt to review early ABR literature primarily due to excellent reviews that are already published. The interested reader, however, would find Downs (13) very thorough.

The current literature pertinent to ABR testing of neonates and NICU populations is discussed and from several aspects (5$7,11,12,17,20,25,34,36,47)$. Weber (47) and Cox et al. (5, 6) have reported norms while Mjoen (32), Hecox et al. (20), Horning (24), and Cox et al. (7) have explored test reliability. The influence of various factors on the ABR have been explored by Barden and Peltzman (2), Bernard et al. (4), Galambos and Despland (15), Kileny et al. (27), Marshall et al. (29), Hecox and Cone (21), Hecox et al. (20), Cox et al. (8), and Roberts et al. (35). ABR as a screening tool has been reported by Horning (24), Crowell et al. (9), Hecox et al. (20), and Salamy et al. (37), while Simmons (43) and Galambos et al. (17) have compared ABR with Crib-O-Gram testing. These citations illustrate some of the current literature that is pertinent to neonatal ABR testing. In the following sections, specific aspects are discussed separately.

\section{TEST PROTOCOL}

The ABR protocol has undergone many refinements, a number of which have been facilitated by advances in commercial equipment. Factors considered in discussing current protocols include placement of the electrode, selection of intensity levels, test environment, state of the neonate, nature of the stimulus, and rate of presentation.

The most popular electrode configuration has usually been vertex $(\mathrm{Cz})$ active with ipsilateral mastoid or earlobe reference and contralateral mastoid or earlobe as ground $(2,4,9,12,20$, $27,34,36$ ). With possible fontanel problems, however, several authors have found forehead placement of the active electrode instead of the vertex to be effective and simple $(7,18,25,28$ $30,32,33,35$ ).

Intensity levels are typically designated as normal hearing level (as before a jury of normal listeners), hearing level (corresponds to manufacturer's dial reading), sound pressure level $(0.0002$ dyne $/ \mathrm{cm}^{2}$ ), sensation level (amount of intensity above individual threshold), and peak equivalent (equivalent amplitude of pure tone on oscilliscopic display). For simplicity sake, normal hearing level is best to use with periodic rechecks either behaviorally or with sound pressure level measure once normal hearing level has been determined. 
The test environment and condition of the subject are important and can influence the results. Generally ABR testing (screening) can be done in the nursery. If testing occurs in the NICU, it is important that it be done when the neonate is stable (in room air, free from respiratory disturbances, etc.). Testing can occur in other areas in the NICU and when the subject is acutely ill, but as will be discussed later, the reliability of the procedure is reduced. If the infant is attached to monitors, IVs, etc., some electrical interference can occur and may require temporary shutdown of this equipment or repositioning the subject. Ambient noise can also be a problem due to differential effects on the various waveform components (44). Early ABR components (Wave I) do not appear to be affected by noise while the later components (Wave V) exhibit increased latencies (45). It is desirable when testing in the NICU to obtain ABR data at low levels of ambient noise. This on occasion may require testing in an adjacent room.

Stimuli typically consist of clicks of 100 -msec duration. Although click polarity can affect waveform latency and morphology (45), the literature is unresolved with regards to preference for condensation or rarefaction polarity (22). It has been shown, however, that very adquate waveform reproduction can be obtained with alternating polarity clicks and in addition there is a reduction in stimulus artifact $(6-8,25)$.

The rate of stimulus presentation varies from $10(25)$ to 38 (47) per sec. With faster presentation rates, peak definition is degraded, Wave I amplitude is reduced, and ABR threshold may be increased in premature infants (8). A good compromise between test speed and wave identification is $21 / \mathrm{sec}$ which is generally satisfactory (28).

Normative data. Several studies have reported normative values for neonates $(5,6,11,15,22,25,28,45,47)$. The general purpose has been to determine what the expected responses are in order that abnormal or atypical findings become apparent. However, a caveat must be issued if one expects to use the published norms for their particular equipment, environment, and population: a considerble number of individual or combined factors are unique to each test setting. These factors interact to influence a response such that each setting will have different normal response values. Published norms can be used for comparison purposes but according to Weber (47) not transposed as exact values to other test settings. Therefore, it is imperative that each setting determine its own normative data.

Risk factors. A number of perinatal events are thought to be associated with hearing loss, e.g., low birth weight, hyperbilirubinemia, asphyxia, acidosis, aminoglycoside drug therapy, intracranial hemorrhage, hyaline membrane disease. Several studies have been published which have attempted to isolate these individual factors and correlate their respective effects on auditory function via the ABR response, $(2,8,15,20,21,27,29$, 35). As of yet, however, relationships between ABR-determined hearing impairment and specific risk factors are tenuous at best. If combined factors are looked at, however, they appear to be highly predictive of ABR-determined impairment (8). In essence, the more risk factors involved, the more likely the neonate will have abnormal $A B R$ results.

Screening. The American Academy of Pediatrics Joint Committee on Infant Hearing published their most recent position statement in September 1982 (1). Within the statement, the use of behavioral or electrophysiologic procedures was recommended in early identification of hearing impairment. Studies detailing ABR as a screening tool $(9,14,24,37,39)$ have generally reported that it meets the screening criteria and is a reliable tool.

The screening procedure typically consists of testing in the nursery at two levels of intensity: one high and one low (60-70 and $30-40 \mathrm{~dB}$ ). Failure criteria consist of absence of a response at either intensity. Recently, Roberts et al. (35) suggested that $50 \%$ of neonates may fail at $40 \mathrm{~dB}$. This would suggest that a $40-\mathrm{dB}$ level is somewhat insensitive in identifying normal versus abnormal function. However, Weber (47) found consistent re- sponses at $30 \mathrm{~dB}$ in 130 of 200 subjects and Lee and Cox (28) found consistent responses at $40 \mathrm{~dB}$ in $100 \%$ of their subjects. Although a 40-dB level can be used, it decreased the sensitivity of the test (47). Screening levels of 30 and $60 \mathrm{~dB}$ appear to be most efficient.

Failures from the screening should be referred for follow-up testing while additional testing of neonates who pass the screening is unwarranted. There are however, exceptions to this format, e.g., subsequent intracranial hemorrhage or meningitis, etc. The time period for follow-up testing of failures is critical. Maturational changes and resolution of transient problems may not be seen if retesting occurs too soon, thereby prolonging a high false positive rate. On the other hand, if retesting is delayed too long, valuable time that is critical for habilitation will be lost. The optimum time for ABR retesting appears to be at 3-4 months of age. A word of warning is in order: subsequent ABR testing in isolation is hazardous. Behavioral and impedance testing should be used in concert with ABR to determine auditory function (6, 46, 48).

Reliability. Of all aspects associated with ABR testing, reliability is perhaps one of the least documented and, as of late, most questioned. A major difficulty with determining the reliability of ABR testing is that the auditory system is not mature at birth and affects the ABR response commensurately. Coupled with the ongoing maturation are the various factors discussed previously which may affect the ABR response when occurring but not after resolution.

The incidence of reported NICU ABR abnormality is approximately $15 \%(17,24,32)$. Subsequent retesting after hospital discharge produces considerably lower figures, i.e., $2-8 \%(7,15)$. The reported incidence of substantial hearing loss in the same population appears to be around $2 \%(17,39,42)$. The false positive rate, then, may approximate $13 \%$.

This per cent difference between test-retest reflects what appears to be a false positive rate which may lead to questioning the reliability of $A B R$. If one compares ABR with other screening procedures like COG, the false positive rate does not seem unusual. For example, Wright and Rybak (49) reported the false positive rate for COG in a NICU population was $80 \%$. With new test procedures, more experience, and close attention to test environment and status of the subject, the false positive rate could realistically be reduced to a single digit percentage. For example, Hooks and Weber (23) report that with bone conduction stimuli the overall failure rate with ABR in the NICU is 2$3 \%$. This failure rate approximates the incidence of hearing loss in the NICU population.

Although the false positive rate may appear to be relatively high, the false negative rate is not known. As near as the present writer is able to determine, however, a single false negative case is yet to be firmly identified.

The initial ABR failures which subsequently pass may indeed reflect abnormality at the time of initial testing. These abnormalities may be related to problems in later development, particularly those aspects associated with learning. If this is demonstrated to be the case, then early ABR data could have predictive significance for later development.

To abandon ABR testing at this time because questions of reliability have not as yet been resolved would be foolishly premature, especially since other available contemporary procedures are not better and certainly are not as sensitive as ABR, e.g., identifying unilateral loss or mild to moderate hearing loss $(8,17,49)$. In view of the alternatives to ABR testing, and the potential of assessment that does exist, continued ABR testing in risk and NICU populations is certainly warranted as are follow-up studies to further establish the $\mathrm{ABR}$ as a routine procedure.

There are other reasons not to abandon ABR testing but they are less concrete although further research may demonstrate applicability. Many congenital or perinatal metabolic and neurologic disorders can produce changes that affect the developing 
brain. Since the ABR is sensitive to structural integrity, early diagnosis and lesion localization may be facilitated by ABR testing (44).

A point needs to be discussed regarding ABR versus other procedures like the COG or the Linco-Bennett auditory response cradle. These seem to be the only two types of procedures available for NICU or nursery testing and it appears that a choice has to be made between the two because they are not compatible. That assumption is incorrect. The COG and response cradle procedures measure the number of physical responses a neonate makes to a loud stimuli $(3,30,40)$. Information for separate ears is not obtainable and mild and moderate hearing loss can be missed. The factors which make the COG and response cradle attractive are cost and time. A large number of neonates can be tested in a short time relatively inexpensively.

The ABR procedure is different from the COG and response cradle in that the response is electrophysiologic in nature. Information about separate ears, neurologic impairment, and hearing sensitivity can be obtained (17). The major stumbling block to universal use has been cost of equipment and trained test personnel. The equipment cost at present is less prohibitive than in recent years, however, Manufacturers are now designing and producing equipment that is smaller in size, considerably lower in price and yet very adequate for ABR testing in the NICU. The trained personnel shortage is less acute since training programs are now incorporating ABR coursework in their curricula and with the widespread growth of ABR in the NICU, practicum and training sites are now available. In the very near future, the major stumbling blocks mentioned previously may well be eliminated.

Currently there is an alternative approach to the problem of cost and test personnel. Davis (10) and others (33, 37) have reported that the Infant Hearing Assessment Program is a means whereby neonates can be tested with $\mathrm{ABR}$ at a minimal cost. The Infant Hearing Assessment Foundation is a nonprofit organization devoted to identifying hearing loss in infants (37). The foundation provides both equipment and test personnel to the hospital which makes inexpensive ABR testing feasible. As of yet, however, the validity of the procedure and the reliability of equipment are yet to be established in referred publications.

In the literature reviewed for the current article, an overwhelming majority of the articles have been favorable in regard to NICU and at-risk neonate testing. This does not necessarily validate the ABR procedure since the literature has not been unanimous in support of neonatal ABR testing $(13,35,43)$. While a difference in opinion is healthy, a closer look at the rationale presented by those opposed would be helpful in a final judgment regarding ABR use.

Downs (13) in his review of ABR has suggested a cautious approach to neonatal ABR testing. The present writer is certainly in agreement and would strongly reinforce caution in ABR testing. With experience, however, this writer and those who have been using ABR with neonates for a similar length of time, have found that the procedure has been reliable and predictive of auditory disorder.

In response to Roberts et al. (35) who questioned the effectiveness of neonatal ABR, their data warrant close inspection. The average age of the infants at the time of ABR testing was 30 weeks gestation. Many of the infants were acutely ill and still on assisted ventilation. Very few screening programs follow this practice. In general, ABR testing is done just prior to discharge when the infant is in a room and free from respiratory disturbances (5). Testing accomplished when infants are still very young and before they have stabilized can affect the ABR results (8) and is ill-advised.

Also, out of an initial test population of 75 subjects, 44 were classified as ABR failures. Of these 44 failures, only 10 were actually subsequently tested. To make conclusions regarding the effectiveness of a procedure with such limited follow-up testing is fallacious. Furthermore, Davis, the second author of Roberts et al., has stated that $\mathrm{ABR}$ is an effective tool for neonatal testing (10).
Simmons (43) criticizes neonatal ABR use because of the practice of labeling initial failures $(15 \%)$ as being hearing impaired. The present writer is certainly in agreement with this but would advise not to discontinue ABR testing, but to discontinue the labeling. The determination or pronouncement of hearing impairment should not be made until follow-up ABR and behavioral testing has occurred. In fact, it has been the experience of this writer that determination of a hearing impairment and the subsequent fitting of a hearing aid before 4 months of age is at best tenuous.

\section{SUMMARY}

Although neonatal ABR testing is not without opposition, the majority of the literature supports its use. If a cautious approach is used, i.e., testing when the infant is stable, monitoring environmental conditions, obtaining individual norms, and allowing only qualified personnel (trained and experienced) to test, the ABR procedure can be a powerful tool in positively identifying hearing loss at a very early age. The ABR procedure is by no means perfected at this point and additional research is ongoing. In the opinion of this writer, however, additional research will ony add to the sensitivity and diagnostic power of the test.

\section{REFERENCES AND NOTES}

1. American Academy of Pediatrics Position Statement 1982 Pediatrics $70: 496$

2. Barden TP, Peltzman P 1980 Newborn brain stem auditory evoked responses and perinatal clinical events. Am J Obstet Gynecol 136:912

3. Bennett MJ 1979 Trails with the auditory response cradle: neonatal response to auditory stimuli. Br J Audiol 13:125

4. Bernard PA, Pechere JC, Hebert R 1980 Altered objective audiometry in aminoglycosides-treated human neonates. Arch Oto-Rhino-Laryngol 228:205

5. Cox LC, Hack M, Metz DA 1981 Brainstem-evoked response audiometry: normative data from the preterm infant. Audiology 20:53

6. Cox LC, Hack M, Metz DA 1981 Brainstem evoked response audiometry in the premature infant population. Int J Pediatr Otorhinolaryngol 3:213

7. Cox LC, Hack M, Metz DA 1982 Longitudinal ABR in the NICU infant. Int J Pediatr Otorhinolaryngol 4:225

8. Cox LC, Hack M, Metz DA 1984 Auditory brainstem response abnormalities in the very low birthweight infant: incidence and risk factors. Ear Hearing $5: 47$

9. Crowell DH, Pang-Ching G, Anderson RE, Kapuniai LF, Teruya K, Doo G, Wright P, Stephens JK 1980 Auditory screening of high risk infants with brainstem evoked responses and impedance audiometry. Hawaii Med J $39: 277$

10. Davis H 1982 Auditory evoked potentials. Paper presented at Second International Evoked Potentials Symposium, Cleveland, OH, October 18, 1982, Cleveland Clinic Foundation, vol 1

11. Despland PA, Galambos R 1980 Use of the auditory brainstem responses by prematures and newborn infants. Neuropediatrie 11:99

12. Despland PA, Galambos R 1980 The auditory brainstem response (ABR) is a useful diagnostic tool in the intensive care nursery. Pediatr Res 14:154

13. Downs DW 1982 Auditory brainstem response testing in the neonatal intensive care unit: a cautious approach. Asha 24:1009

14. Finitzo-Hieber T, Hecox K, Cone B 1978 Clinical applicability of the auditoryevoked response in nenonates. ORL 4:638

15. Galambos R, Despland PA 1980 The auditory response (ABR) evaluates risk for hearing loss in the newborn. Pediatr Res 14:159

16. Galambos R, Hicks G, Wilson MJ 1982 Hearing loss in graduates of a tertiary infant care nursery. Ear Hearing 3:87

17. Galambos R, Hicks G, Wilson MJ 1982 Identification audiometry in neonates: a reply to Simmons. Ear Hearing 3:188

18. Grundfast KM, Fria TJ, Bartling V, Sylvan ES 1978 Clinical applications of brainstem auditory electric response testing at a children's hospital. Trans Penn Acad Opthalmol Otol 31:205

19. Hack M, Fanaroff AA, Markatz IA 1979 The low-birth-weight infant--evaluation of a changing outlook. N Engl J Med 301:1162

20. Hecox KE, Cone B, Blaw ME 1981 Brainstem auditory evoked response in the diagnosis of pediatric neurologic diseases. Neurology 31:832

21. Hecox KE, Cone B 1981 Prognostic importance of brainstem auditory evoked responses after asphyxia. Neurology 31:1429

22. Hecox KE, Burkard R 1982 Developmental dependencies of the human brainstem evoked response. Ann NY Acad Sci 388:538

23. Hooks RG, Weber BA 1983 Auditory brain stem responses of premature infants to bone conducted stimuli: a feasibility study. Ear Hearing 5:42

24. Horning JK 1979 High risk registry with brainstem auditory evoked response follow-up. Hearing Aid J 32:7

25. Jacobson JT, Morehouse CR, Johnson MJ 1982 Strategies for infant auditory brain stem response assessment. Ear Hearing 3:263

26. Jewett DL, Romano MN, Williston JS 1970 Human auditory evoked potentials: possible brainstem components detected from the scalp. Science 
$167: 1517$

27. Kileny P, Connelly C, Robertson L 1980 Auditory brainstem responses in perinatal asphyxia. Int J Pediatr Otorhinolaryngol 2:147

28. Lee SP, Cox LC 1982 Latency-intensity functions of the auditory brainstem evoked response in premature infants. Florida Language Speech Hearing Association J 3:15

29. Marshall RE, Reichert TM, Kerley SM, Davis H 1980 Auditory functions in newborn intensive unit patients revealed by auditory brainstem potentials. J Pediatr 96:731

30. McFarland WH, Simmons FB, Jones FR 1980 An automated hearing screening technique for newborns. J Speech Hearing Dis 4:495

31. Ment LR, Scott DT, Ehrenkranz RA, Rothman SG, Duncan CC, Warslaw WB 1982 Neonates of $<1,250$ grams birthweight; prospective neurodevelopmental evaluation during the first year post-term. Pediatrics 70:292

32. Mjoen S 1981 ABR in pediatric audiology. Scand Audiol Suppl 13:141

33. Morganstern N 1982 An infant hearing assessment program. Hearing Inst 33:44

34. Morgan A, Salle B 1980 A study of brainstem evoked responses in prematures. Acta Otolaryngol 89:370

35. Roberts JL, Davis H, Phon GL, Reichert TJ, Sturtevant EM, Marshall RE 1982 Auditory brainstem responses in preterm neonates: maturation and follow-up. J Pediatr 101:251

36. Salamy A 1981 The theoretical distribution of evoked brainstem activity in preterm, high-risk, and healthy infants. Child Dev 52:752

37. Salmy A, Somerville G, Patterson D 1982 The infant hearing assessment program. Hearing Aid J 35:10

38. Schulman-Galambos C, Galambos R 1975 Brainstem auditory-evoked responses in premature infants. J Speech Hearing Res 18:456
39. Schulman-Galambos C, Galambos R 1979 Brainstem evoked response audiometry in newborn hearing screening. Arch Otolaryngol 105:86

40. Shepard NT 1983 Newborn hearing screening using the Linco-Bennett Auditory Response Cradle: a pilot study. Ear Hearing 4:5

41. Simmons FB 1978 Identification of hearing loss in infants and young children. Otolaryngol Clin N Am 11:19

42. Simmons, FB, McFarland WH, Jones FR 1979 An automated hearing screening technique for newborns. Act Otolaryngol 87:1

43. Simmons FB 1982 Comment on "hearing loss in graduates of a tertiary intensive care nursery." Ear Hearing 3:188

44. Sohmer H 1983 Neurologic disorders. In: Moore EJ (ed) Bases of Auditory Brain-stem Evoked Responses. New York, Grune and Stratton, pp 317-341

45. Stockard JE, Westmoreland BF 1981 Technical consideration in the recording and interpretation of the brainstem auditory evoked potential for neonatal neurological diagnosis. Am J EEG Technol 21:31

46. Stockard JE, Stockard JJ 1983 Recording and analyzing. In: Moore EJ (ed) Bases of Auditory Brain-stem Evoked Responses. New York, Grune and Stratton, pp 255-286

47. Weber BA 1982 Comparison of auditory brainstem response latency norms for premature infants. Ear Hearing 3:257

48. Worthington DW, Peters J 1980 Quantifiable hearing and ABR: paradox or error? Ear Hearing 5:281

49. Wright LB, Rybak LP 1983 Accuracy of Crib-O-Gram as screening test on high risk newborns. [Suppl 1] J Acoust Soc Am 73:79

50. Address correspondence to Dr. L. Clarke Cox, Department of Audiology, Cleveland State University, Cleveland, $\mathrm{OH} 44115$.

51. Received for publication August 15, 1983.

\title{
Lipid Profiles and Lipase Activities in Children and Adolescents with Chronic Renal Failure Treated Conservatively or with Hemodialysis or Transplantation
}

\author{
KOHTARO ASAYAMA, ${ }^{(26)}$ HIROSHI ITO, CHIEKO NAKAHARA, AKIRA HASEGAWA, AND \\ KIYOHIKO KATO \\ Department of Pediatrics, School of Medicine, Keio University [K.A.] and Department of Pediatrics and Urology, \\ Tokyo Metropolitan Children's Hospital [H.I., C.N., A.H.], Tokyo, Japan and Department of Pediatrics, \\ Yamanashi Medical College [K.K.], Yamanashi, Japan
}

\section{Summary}

To elucidate the pathogenesis of hyperlipidemia in chronic renal disease in children and adolescents, we have measured serum triglyceride, total cholesterol, high density lipoprotein cholesterol (HDL-C) and activities of postheparin plasma lipoprotein lipase and hepatic triglyceride lipase (EC 3.1.1.3) in nine patients with transplants, and nine hemodialyzed and 18 conservatively treated patients with chronic renal failure. In 29 of 36 patients, serum insulin levels both in fasting and in response to oral glucose load were measured. The lipase activities were measured separately, utilizing antiserum against hepatic triglyceride lipase.

All groups of patients had hypertriglyceridemia. The patients with endogenous creatinine clearance less than $20 \mathrm{ml} / \mathrm{min} / \mathrm{m}^{2}$ had a low HDL-C level. The HDL-C level was correlated inversely with serum triglyceride level and positively with glomerular filtration rate. The lipoprotein lipase activities were low in patients with endogenous creatinine clearance less than $20 \mathrm{ml} / \mathrm{min} /$ $\mathrm{m}^{2}$. Although hepatic triglyceride lipase activities were not significantly low in any groups of patients, they were correlated with glomerular filtration rates in the conservatively treated patients with chronic renal failure. A defective triglyceride removal due to low lipase activities may contribute to uremic hypertriglyceridemia in these patients. On the other hand, patients with transplants had almost normal lipase activities and exhibited hyperinsulinemia; overproduction of triglyceride due to hyperinsulinemia may contribute to their hypertriglyceridemia.

\section{Abbreviations}

LPL, lipoprotein lipase

HTGL, hepatic triglyceride lipase

HD, hemodialysis

CRF, chronic renal failure

Tx, transplant

TG, triglyceride

TC, total cholesterol 